\title{
Estudo Comparativo da Atividade Antimicrobiana da Ciprofloxacina, Gentamicina e Ceftriaxona
}

\section{Angelma Genaro e Carlos Alberto de Magalhães Lopes}

E. coli é a causa mais comum de infecção urinária, sendo responsável por $90 \%$, ou mais, das infecções adquiridas na comunidade, onde sua resistência a diversas drogas tem sido motivo de preocupação das autoridades. O trabalho teve como objetivo comparar a eficácia da ciprofloxacina, ampicilina e ceftriaxona "in vitro" na forma de medicamento de referência e de medicamento genérico. Foram obtidas 79 linhagens provenientes de urina de pacientes com infecção urinária internados no Hospital das Clínicas da Faculdade de Medicina de Botucatu, UNESP, no período de fevereiro a abril de 2004. Foi analisado a suscetibilidade in vitro através da Determinação da Concentração Inibitória Mínima (CIM). Os resultados demonstraram que a ciprofloxacina e a ceftriaxona não apresentaram diferenças significativas entre os medicamentos genéricos e de referência ao contrário do que foi observado com a ampicilina. Esses dados mostram a eficácia dos medicamentos genéricos quando comparados com os de referência.

Palavras-chave: ciprofloxacina; gentamicina; ceftriaxona; atividade antimicrobiana.

E. coli is considered the main etiological agent in the urinary tract and its resistance to several drugs has been a reason of preoccupation of the authorities. The objective of this work was to compare the antimicrobial commercial drugs effectiveness in its generic and brand mark presentations. 79 strains isolated from patients with urinary infection and interned at the Hospital das Clinicas ( School of Medicine -Botucatu - UNESP), during the period of February to April of 2004. It was in vitro analyzed the ceftriaxone, ciprofloxacin and ampicilin susceptibility through determination of the Minimum Inhibitory Concentration (MIC). The results showed that the ciprofloxacin and ceftriaxone generic, as well as the brand mark drugs presented the same effectiveness. In general and according to our observations it is suggestive that the antimicrobial effectiveness of the generic drugs still demand new and wider investigations especially concerning the detections of possible discrepancies with brand mark products of industrial sections showing lower technological development level.

Keywords: ciprofloxacin; gentamicin; ceftriaxone; antimicrobial activity. 


\section{Introdução}

As bactérias da Família Enterobacteriaceae constituem um grande grupo heterogêneo de bastonetes Gram negativos, cujo hábitat natural é o trato intestinal de humanos e animais ${ }^{1}$. A família inclui numerosos gêneros e a espécie $E$. coli é constituída por uma variedade relativamente grande de linhagens patogênicas, sendo capaz de causar infecções intestinais, infecções urinárias, sepses, meningites e outros tipos de infecções ${ }^{2}$.

O microrganismo é a causa mais comum de infecção urinária, sendo responsável por $90 \%$, ou mais, das infecções adquiridas na comunidade. $\mathrm{O}$ tratamento da infecção é realizado através do uso de antibacterianos, porém o uso indiscriminado dessas drogas levou ao aumento na resistência dessas bactérias a vários antimicrobianos $^{3}$. As fluoroquinolonas constituem uma importante classe de drogas para o tratamento de infecções do trato urinário e Isto ocorre, em grande parte, devido a sua excelente atividade contra a E. coli. ${ }^{4,5,6}$. Os antibióticos $\beta$-lactâmicos, por sua vez, estão entre os antimicrobianos mais freqüentemente prescritos no mundo todo, constituindo a emergência de resistência a esses agentes, uma decorrência nas duas últimas décadas, da pressão seletiva exercida ao incremento de seu uso terapêutico? ${ }^{7}$.

O tratamento das infecções e de outras doenças no âmbito hospitalar e na comunidade gera um custo altíssimo para o governo e para a população e com o objetivo de tornar o tratamento medicamentoso mais acessível a todas as classes sociais, foi legalizado no Brasil o medicamento genérico.

Como determina a lei que regulamenta a comercialização desses medicamentos no Brasil, antes de chegarem ao mercado, os produtos devem passar pelos testes de biodisponibilidade e de bioequivalência para que seja verificado se possuem eficácia igual à do medicamento de marca. Contudo, apesar do avanço das pesquisas na área dos antimicrobianos, ainda não existem na bibliografia informações que comprovem a eficácia dos medicamentos genéricos através de testes in vitro em relação à dos medicamentos de marca ${ }^{8}$. Tendo em vista esta problemática, o presente trabalho teve como objetivo comparar a eficácia da ceftriaxona, ciprofloxacina e ampicilina in vitro nas formas de medicamento de marca e de genérico.

\section{Materiais e Métodos}

\section{LINHAGENS}

Foram estudadas 79 amostras de Escherichia coli isoladas de amostra de urina, no período de fevereiro a abril de 2004, de pacientes do Hospital das Clínicas (HC) da Faculdade de Medicina de Botucatu, UNESP, Botucatu com infecção no trato urinário. As linhagens foram identificadas pelo laboratório de Análises Clínicas do $\mathrm{HC}$ e posteriormente transportadas para o laboratório de Microbiologia do Departamento de Microbiologia e Imunologia do Instituto de Biociências, Campus de Botucatu-UNESP.

\section{DROGAS ANTIMICROBIANAS}

Droga genérica: Ampicilina (Eurofarma), Cloridrato de Ciprofloxacina (EMS) e Ceftriaxona Sódica (Eurofarma). Droga de marca ${ }^{\circledR}$ : Amplacilina (Eurofarma), Cipro (Bayer) e Rocefin (Roche).

\section{DETERMINAÇÃO DA CONCENTRAÇÃO INIBITÓRIA MÍNIMA (CIM)}

A sensibilidade antimicrobiana das linhagens frente a ciprofloxacina, ampicilina e ceftriaxona genérica e de marca $^{\circledR}$, foi avaliada através da Determinação da Concentração Inibitória Mínima, pela diluição da droga em Ágar, conforme as normas estipuladas pelo National Committee for Clinical Laboratory Standards-NCCLS 9 (2004). (Tabela 1).

\section{ANÁLISE ESTATÍSTICA}

Os dados foram analisados estatisticamente através do teste de Goodman ${ }^{10,11}(1964,1965)$ do teste de Norman $\&$ Streiner $^{12}$ (1999).

\section{RESULTADOS}

Os resultados mostram que a ceftriaxona foi à droga mais efetiva com um perfil de sensibilidade de $93,7 \%$ e $92,4 \%$ para a droga genérica e de marca 
respectivamente, nas 79 linhagens de Escherichia coli estudadas. Foi observada a presença de seis linhagens de E. coli sensíveis a ampicilina genérica, porém resistentes a ampicilina marca ${ }^{\circledR}$ e uma linhagem sensível a ciprofloxacina de $\operatorname{marca}^{\circledR}$, porém resistente a ciprofloxacina genérica. (Tabela 2)

Os mais elevados percentuais de perfis de concordância para a sensibilidade das linhagens para ambas as drogas genérica e de marca $^{\circledR}$ foram caracterizados para a ceftriaxona e de resistência para a ampicilina. (Figura 1)

Tabela 1: Padronização para a interpretação das Concentrações Inibitórias Mínimas -CIM $(\mu \mathrm{g} / \mathrm{mL})$.

\begin{tabular}{|c|c|c|c|}
\hline Drogas & Resistente & Sensível & Intermediário* $^{*}$ \\
\hline Ciprofloxacina & $\geq 4$ & $\leq 1$ & 2 \\
\hline Ceftriaxona & $\geq 64$ & $\leq 8$ & $16-32$ \\
\hline Ampicilina & $\geq 32$ & $\leq 8$ & 16 \\
\hline
\end{tabular}

Nota:

National Committee for Clinical laboratory Standards-NCCLS ${ }^{34}(2004)$

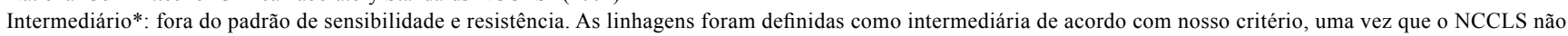
apresenta esta categoria.

Tabela 2: Distribuição percentual das 79 linhagens de Escherichia coli segundo os perfis de sensibilidade obtidos para cada droga na forma de medicamento genérico e de marca ${ }^{\circledR}$ em relação ao teste de Determinação da Concentração Inibitória Mínima.

\begin{tabular}{|c|c|c|c|c|c|}
\hline Drogas & & $S n^{\circ}(\%)$ & $\mathrm{R} \mathrm{n}^{0}(\%)$ & I $n^{0}(\%)$ & Total $n^{0}(\%)$ \\
\hline & Genérico & $74(93,7)$ & $3(3,8)$ & $2(2,53)$ & $79(100)$ \\
\hline Ceftriaxona & $\operatorname{Marca}^{\circledR}$ & $73(92,4)$ & $5(6,3)$ & $1(1,3)$ & 79 (100) \\
\hline & Genérico & $54(68,4)$ & $25(31,6)$ & $0(0,0)$ & $79(100)$ \\
\hline Ciprofloxacina & $\operatorname{Marca}^{\circledR}$ & $55(69,6)$ & $24(30,4)$ & $0(0,0)$ & $79(100)$ \\
\hline & Genérico & $26(32,9)$ & $49(62,0)$ & $4(5,6)$ & $79(100)$ \\
\hline Ampicilina & Marca $^{\circledR}$ & $18(22,8)$ & $57(72,1)$ & $4(5,1)$ & 79 (100) \\
\hline
\end{tabular}

Nota:

S: sensível; R: resistente; I: intermediário; G: genérico; M: marca; CROG,M: ceftriaxona genérica e de marca;

CIPG,M: ciprofloxacina genérica e de marca; AMPG,M: ampicilina genérica e de marca 


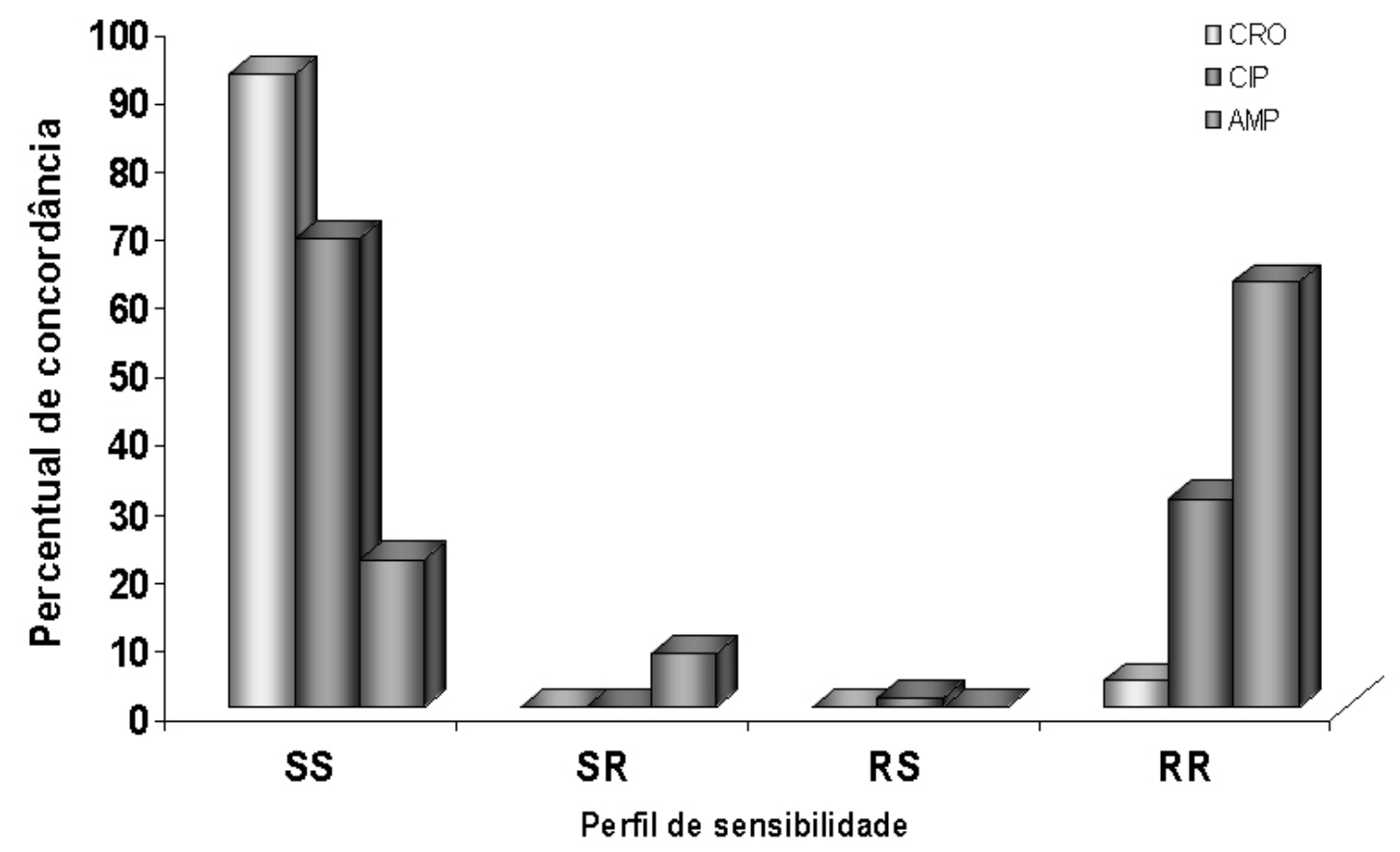

Nota:

SS: perfil único de sensibilidade para a droga nas formas genérica e de marca.

SR: perfil de sensibilidade e de resistência para a droga nas formas genérica e de marca, respectivamente.

RS: perfil de resistência e sensibilidade para a droga nas formas genérica e de marca, respectivamente.

RR: perfil único de resistência para a droga nas formas genérica e de marca.

CRO: ceftriaxona; CIP: ciprofloxacina; AMP: ampicilina.

Figura 1: Distribuição percentual da concordância entre as drogas genérica e de marca ${ }^{\circledR}$.

\section{Discussão}

Segundo os dados do Ministério da Saúde (MS), dos 170 milhões de brasileiros 50 milhões estão excluídos do acesso aos medicamentos e de qualquer tipo de assistência médica ${ }^{13}$. Os dados da Organização Mundial da Saúde (OMS) são coincidentes, pois se estima que um terço da população mundial também não tenha acesso a medicamentos.

Diante deste contexto, a própria OMS definiu os princípios para as Políticas Nacionais de Medicamentos, visando melhorar o acesso e promover uso racional de medicamentos ${ }^{14}$. Entre as estratégicas propostas, os medicamentos genéricos constituem parte essencial, uma vez que representam uma alternativa para assegurar a disponibilidade de medicamentos de qualidade comprovada e baixo custo ${ }^{15,16}$.

O Brasil seguindo as diretrizes mundiais definiu as bases legais para a instituição do medicamento genérico em 10 de fevereiro de 1999, com a aprovação da Lei $n^{\circ}$ 9787/99, que ficou conhecida como "Lei dos Genéricos" $" 17,18$.

Hoje existem 1.700 medicamentos genéricos legalizados no Brasil, mostrando assim o grande crescimento na indústria dos genéricos. Embora sejam realizados testes de equivalência farmacêutica, existem poucos trabalhos científicos a respeito da efetividade dessas drogas. Dentre tanto medicamentos genéricos legalizados no Brasil, vários representam uma classe extremamente importante na terapêutica, uma vez que a utilização de drogas de marca representa um elevado 
custo para o Estado e para a população.

Através da análise dos nossos resultados, verificase que não houve diferenças significativas entre a ação das drogas em sua forma genérica e de marca. Porém um dado importante a ser considerado é que a análise da sensibilidade antimicrobiana permitiu-nos observar a presença de seis linhagens sensíveis a ampicilina genérica, porém resistentes a ampicilina de marca $^{\circledR}$. O mesmo foi observado para a ciprofloxacina, onde uma linhagem de Escherichia coli apresentou perfil de sensibilidade para a droga marca e resistência para a droga genérica.

Estes resultados, pela usa importância, no que diz respeito à diferença da efetividade da droga na forma genérica e de marca ${ }^{\circledR}$, demonstra a necessidade de mais estudos para elucidar melhor a qualidade destas drogas. Infelizmente, a ausência de bibliografia especializada não permite uma análise mais apurada dos resultados, o que seria de grande interesse aos clínicos e à comunidade científica. Nossos resultados sobre os percentuais de sensibilidade de linhagens de Escherichia coli através da difusão da droga a partir de discos impregnados, permitiram-nos ainda observar a excelente atividade da ceftriaxona $(97,5 \%)$ e da ciprofloxacina $(79,8 \%)$ frente às linhagens estudadas.

Através dos resultados alcançados, consideramos importante ressaltar a necessidade novas investigações através de outras metodologias e que apesar das drogas na forma de genéricos terem apresentado uma atividade semelhante às de marca ${ }^{\circledR}$, não podemos esquecer que existem inúmeros genéricos no mercado produzidos por diferentes laboratórios farmacêuticos, onde a qualidade e a eficácia ainda surgem como dúvida para os clínicos e consumidores em função das diferenças de qualidade no processo tecnológico de sua produção.

\section{Conclusões}

Os resultados da sensibilidade antimicrobiana demonstraram um melhor desempenho da ceftriaxona em relação ao da ciprofloxacina e da ampicilina, frente às linhagens de Escherichia coli. As drogas genéricas foram tão efetivas quanto as drogas de marca quanto à eficiência antimicrobiana.

\section{REFERÊNCIAS}

1. Murray, P.R.; Baron, E.J.; Pfaller, M.A.; Tenover, F.C.; Yolken, R.H.; Manual of clinical microbiology. 6nd ed., Washington: American Society for Microbiology; 1995.

2. Trabulsi, L.R.; Alterthum, F.; Gompertz, O.F.; Candeias, J.A.N.; Microbiologia. São Paulo: Atheneu; 1999.

3. Schor, N. \& Srougi, M. Nefrologia, urologia clínica. São Paulo: Sarvier; 1998.

4. Graninger, W.; Zedtwitz-Liebenstein, K.; Laferl, H.; Burgmann, H. Chemotherapy (Basel). 1996, 42 (Suppl.1), 43-53.

5. Hendersho, E.F.; Fluoroquinolones. Infect Dis Clin North Am. 1995, 9, 715-30.

6. Naber, K.G. J Antimicrob Chemother. 2000, 46 (Suppl A), 23-7.

7. Goodman, L.S.; Gilman, A.; As bases farmacológicas da terapêutica, 9a ed., McGraw Hill: Rio de Janeiro, 1996.

8. Storpirtis, S.; Balduíno, J.; Bueno, M.M.; Freitas, S.T.; Gatto, R.C.; Lima, F.P.; et al. Aspectos técnicos relativos ao registro de medicamento genérico no Brasil. Brasília: Agência Nacional de Vigilância Sanitária; 2003.

9. National Committee for Clinical Laboratory Standards (NCCLS). Performance standards for antimicrobial susceptibility testing: fourteen informational supplement NCCLS, document M100S14, Pennsylvania, USA, 2004.

10. Goodman, L.A. Ann Math Stat. 1964, 35, 716-25.

11. Goodman, L.A. Techonometrics. 1965, 7, 247-54.

12. Norman, G.R.; Streiner, D.L.; Biostatistics: the bore essentials. St. Louis: Mosby Year Book; 1994.

13. Brasil. Ministério da Saúde. Informações em saúde. Disponível em: http://www.saude.gov.br. Acessado em 14/04/2008. 
14. Veiga, F.J.B.; Política de medicamentos: genéricos ou marca registrada? Experiência da União

Européia. In: Resumos do $9^{\circ}$ Congresso da Ordem dos Farmacêuticos Iberolatinos. Salvador. Salvador: OFIL, 2000.

15. Bermude, J.A.Z. Cad. Saúde Pública. 1994, 10, 368-78.

16. World Health Organization. Essential drugs and medicines policy. Disponível em: http://www. who.int.medicines/teams/par/par_objectives.html. Acessado em 10/04/08.

17. Anvisa. Hotside genéricos. Disponível em: http:// www.anvisa.gov.br/hotside/genéricos/index.htm. Acessado em 14/04/08.
18. Brasil. Agência Nacional de Vigilância Sanitária. Lei n. 9.787, de 10 de fevereiro de 1999. Estabelece as bases legais para a instituição do medicamento genérico no país. Brasília; 1999.

\section{Angelma Genaro*1, Carlos Alberto de Magalhães Lopes'}

${ }^{1}$ Universidade Estadual Paulista Julio de Mesquita Filho, Bauru, SP, Brasil. Departamento de Microbiologia e Imunologia - Instituto de Biociências - Botucatu.

*E-mail: angelmagenaro@hotmail.com 